\title{
Efecto de la humedad relativa en la viabilidad de huevos de Neoseiulus sp. y Proprioseiopsis iorgius (Acari: Phytoseiidae) en condiciones de laboratorio
}

\author{
Effect of relative humidity on the viability of Neoseiulus sp. and \\ Proprioseiopsis iorgius (Acari: Phytoseiidae) eggs under laboratory conditions
}

\author{
Vania Ugrinovic ${ }^{1}$, Víctor Tello ${ }^{1 *}$
}

\begin{abstract}
RESUMEN
El objetivo del estudio fue evaluar la tolerancia de los huevos de Neoseiulus sp. y Proprioseiopsis iorgius Karg (Acari: Phytoseiidae) expuestos a diferentes humedades expresadas como humedad relativa (HR, \%) y como déficit de presión de vapor (DVP, hPa) [6,4\% $(29,6 \mathrm{hPa}) ; 9,2 \%(28,8 \mathrm{hPa}) ; 35,0 \%(20,6 \mathrm{hPa}) ; 37,1 \%(19,9 \mathrm{hPa})$ y $80,0 \%(6,3 \mathrm{hPa})]$. Los ensayos se realizaron a una temperatura de $25 \pm 0,5^{\circ} \mathrm{C}$ y un fotoperíodo de $14: 10 \mathrm{~h}$ (L:O). El porcentaje de eclosión de larvas fue medido a las $12 \mathrm{~h}, 24 \mathrm{~h}, 36 \mathrm{~h}, 48 \mathrm{~h}$ y $60 \mathrm{~h}$. Los resultados mostraron una alta tolerancia de los huevos de Neoseiulus sp. a la baja humedad relativa con una humedad de eclosión media (HR50) de 34,5\% (20,6 hPa) no así $P$. iorgius que registró una $\mathrm{HR}_{50}$ de 72,4\% (8,8 hPa). El porcentaje de eclosión total para Neoseiulus sp. fue de $0,0 \% ; 0,0 \% ; 55,2 \% ; 73,3 \% ; 100,0 \%$ según las humedades evaluadas y para P. iorgius solo se obtuvo resultados para el $80,0 \%$ HR y fue de $91,67 \%$ (a humedades entre $6,4 \%$ y $37,1 \%$ sus huevos fueron inviables). Estudios complementarios de Neoseiulus sp. a futuro podrán dar luz sobre su potencial como agente de control biológico de fitoácaros de cultivos en el desierto del norte de Chile.
\end{abstract}

Palabras clave: eclosión, huevos, $\mathrm{HR}_{50}, \mathrm{VPD}_{50}$.

\begin{abstract}
The aim of the study was to evaluate the tolerance of Neoseiulus sp. and Proprioseiopsis iorgius Karg (Acari: Phytoseiidae) eggs exposed to different humidities expressed as relative humidity $(R H, \%)$ and as vapour pressure deficit $(V P D, h P a)[6,4 \%$ $(29,6 \mathrm{hPa}) ; 9,2 \%(28,8 \mathrm{hPa}) ; 35,0 \%(20,6 \mathrm{hPa}) ; 37,1 \%(19,9 \mathrm{hPa})$ y 80,0\% (6,3 $\mathrm{hPa})]$. The tests were conducted at a temperature of $25 \pm 0,5^{\circ} \mathrm{C}$ and a photoperiod of $14: 10 \mathrm{~h}(\mathrm{~L}: \mathrm{D})$. The eclosion percentage of larvae was measured at $12 \mathrm{~h}, 24 \mathrm{~h}, 36 \mathrm{~h}, 48 \mathrm{~h}$ and $60 \mathrm{~h}$. The results showed a high tolerance of Neoseiulus sp. eggs to low relative humidity with an mean hatching humidity (RH50) of 34,5\% (20,6 hPa); instead P. iorgius showed an RH50 of 72,4\% (8,8 hPa). The total eclosion percentage for Neoseiulus $s p$. was 0,0\%; 0,0\%; 55,2\%; 73,3\%; 100,0\% according to evaluated humidities and $\mathrm{P}$. iorgius only showed results for 80,0\% RH and was of $91,67 \%$ (at humidities between 6,4\% and 37,1\% were non-viable eggs). Complementary studies of Neoseiulus sp. in the future may shed light on its potential as a biological control agent of plant-feeding spider mites of crops in the desert of northern Chile.

Key words: eclosion, eggs, $R H_{50}, V P D_{50}$.
\end{abstract}

\section{Introducción}

Los ácaros tetraníquidos son las plagas más importantes de las plantas cultivadas después de los insectos. Estos pequeños arácnidos, ocasionan severos daños a muchos cultivos, sobre todo cuando se trata de elevados niveles poblacionales (Van Leeuwen et al., 2007). La familia Phytoseiidae ha recibido considerable atención en los últimos 45 años debido al potencial de estos ácaros como agentes de control biológico de una amplia gama de plagas agrícolas, como arañitas rojas, trips y escamas (Ghazi y Amano, 2016). Tienen una alta tasa de reproducción, una tasa de desarrollo rápido y una proporción de hembras equivalentes a la de su presa que les permite responder numéricamente a un aumento en la densidad de sus presas y pueden ser masificados fácilmente en laboratorio. En Chile, aproximadamente 48 especies de ácaros fitoseidos han sido asociadas con plantas cultivadas

\footnotetext{
1 Facultad de Recursos Naturales Renovables, Universidad Arturo Prat, Avenida Arturo Prat 2120, Casilla 121, Iquique, Chile.

* Autor por correspondencia: victor.tello@unap.cl
} 
o con malezas. Algunos de estos ácaros se han reportado como un agente de regulación de las poblaciones de ácaros fitófagos de importancia económica, como Tetranychus urticae Koch, Tetranychus cinnabarinus Boisduval, Panonychus ulmi Koch y Panononychus citri McGregor (Tello et al., 2009). Neoseiulus sp. se distribuye sobre diferentes hospederos en la Región de Tarapacá: Medicago sativa Linn., en La Huayca ( $20^{\circ} 26^{\prime}$ $37,4^{\prime \prime} \mathrm{S}, 6^{\circ} 32$ ' 13,5" O), asociado a Tetranychus desertorum Banks; en Bajo Soga (19² 44' 51,6" S, $69^{\circ} 48^{\prime} 25,8^{\prime \prime}$ O) sobre Citrus limon (Linn.) Burm y Poacea; en Colonia Pintados (20 37' 38,8' S, $69^{\circ} 34^{\prime} 18,5^{\prime \prime} \mathrm{O}$ ) sobre gramíneas (Poacea) y en

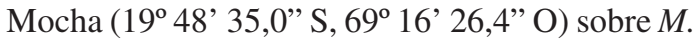
sativa asociado a T. desertorum. Proprioseiopsis iorgius Karg es un ftoseido que fue descrito por Karg (1976) y citado solo para Chile, encontrándose

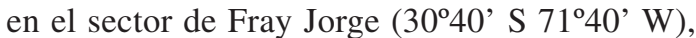
Región de Coquimbo, Chile (Karg, 1976). Tello et al. (2011) extienden su distribución hasta el litoral de la Región de Tarapacá (20¹6'19,3" S, 70 07'51,6" O). Diferentes factores afectan el éxito del control biológico con ácaros de la familia Phytoseiidae, entre los factores abióticos podemos citar la temperatura, la humedad relativa y pesticidas (De Vis et al., 2006; Hua et al., 2014) y dentro de los factores bióticos el canibalismo, la depredación intragremio, la escasez de alimento, la domatia, entre otros (Pozzebon et al., 2015; Ghasemlooa et al., 2016; Guo et al., 2016; Lv et al., 2016). Uno de estos factores, la humedad relativa, afecta directamente sobre la supervivencia de los huevos de los fitoseidos (Walzer et al., 2007; Ghazi y Amano, 2016). Es un gran desafío encontrar depredadores tolerantes a la baja humedad relativa, especialmente para los cultivos donde la humedad es a menudo por debajo del 50-60\%, lo que ha sido considerado como un límite para el desarrollo de muchos de los ácaros depredadores (Ferrero et al., 2010). En los estudios biológicos, las condiciones del vapor de agua se expresan comúnmente como humedad relativa (HR; \%), que es el porcentaje de la presión de vapor [PV; $\mathrm{Pa}$ (Pascales)] en la presión de vapor saturada (PVS; Pa) a una temperatura del aire, o como un déficit de la presión de vapor (DPV; Pa) que es la diferencia entre PVS y PV. Como la pérdida de agua por evaporación desde los organismos es generalmente proporcional al DPV, incluso a diferentes temperaturas del aire, se debería usar el
DPV en lugar de la HR cuando varía la temperatura del aire (Eaton y Kells, 2009).

Estudios efectuados por Van Dinh et al. (1988) en Amblyseius idaeus (Denmark \& Muma) y Amblyseius anonymus Chant \& Baker, revelan que el éxito en la supervivencia de fitoseidos en ambientes de baja humedad relativa $(<60 \%,>12,7 \mathrm{hPa})$ se relacionan directamente con el origen climático de los fitoseidos (los originados en zonas áridas estarían más adaptados a condiciones de baja humedad). Muchos fitoseidos tienen bajos porcentajes de eclosión de larvas, por debajo del 50\% HR. Los huevos son considerados el estadio más sensible a las condiciones de baja humedad, porque los estadios móviles son capaces de reponer el contenido de agua por medio de la alimentación, el consumo de agua libre, o la búsqueda de sitios favorables (Walzer et al., 2007). La capacidad de eclosión de las larvas de los ácaros fitoseidos normalmente muestra una respuesta sigmoidal, siendo mínima a la humedad cercana a cero y máxima a nivel de saturación (Bakker et al., 1993). Existen variaciones en susceptibilidad a las condiciones de baja humedad entre las especies y "strains" que habitan diferentes regiones climáticas. En las especies de zonas húmedas, la eclosión de las larvas a una humedad relativa por debajo del $60 \%$ y temperaturas que van desde 20 a $32^{\circ} \mathrm{C}$ es cero o muy baja, mientras que la eclosión de larvas de especies de zonas áridas ocurre a humedades de alrededor del $30 \%$ y a temperaturas que van de 20 a $25{ }^{\circ} \mathrm{C}$ (Negm et al., 2014). De acuerdo con los antecedentes anteriores se planteó evaluar el efecto de la humedad relativa en la viabilidad de huevos de Neoseiulus sp. y $P$. iorgius en condiciones de laboratorio.

\section{Materiales y Métodos}

\section{Ubicación de los ensayos}

Los bioensayos se realizaron en los laboratorios de la Estación Experimental Canchones (Facultad de Recursos Naturales Renovables) de la Universidad Arturo Prat, ubicada en la Pampa del Tamarugal, Desierto de Atacama, Chile (20²6'39,42” S; 69³2'00,58' O, $995 \mathrm{~m}$ sobre el nivel del mar).

\section{Material biológico}

Neoseiulus sp. fue colectado en grama, Cynodon dactylon (Linneo) (Poaceae), en el oasis de Pica (20²9'12,4”S, 69¹9'33,9”O) y P. iorgius fue 
colectado en rayito de sol, Mesembryanthemum roseum Willd. (Aizoaceae), en la localidad de Iquique ( $\left.20^{\circ} 16^{\prime} \mathrm{S} 70^{\circ} 7^{\prime} \mathrm{W}\right)$, ambos lugares se ubican en la Región de Tarapacá, Chile.

\section{Crianza de Neoseiulus sp. y $P$. iorgius}

Los especímenes colectados se dispusieron en placas negras de acrílico de $10 * 10 \mathrm{~cm}$ para su crianza masiva tal como las descritas por Swirski et al. (1970). Para evitar el escape de los ácaros se adicionó pegamento (Point Sticken Glue ${ }^{\circledR}$, Point Chile S.A.) en el borde de las placas. Las crianzas se realizaron en salas con ambiente controlado bajo condiciones de $25 \pm 0,5^{\circ} \mathrm{C}$ de temperatura, $80 \pm 10 \%$ HR y un fotoperíodo de 14:10 h (L:O). Los fitoseidos fueron alimentados con una dieta mixta de huevos, estados ninfales y adultos de T. desertorum. Los ácaros fitófagos fueron multiplicados en plantas de frejol (Phaseolus vulgaris Linn.), sembrándose 10 semillas por macetero cada 7 días y se mantuvieron en condiciones de $25 \pm 0,5^{\circ} \mathrm{C}$ de temperatura, $70,0 \pm 10 \%$ HR y un fotoperíodo de 14:10 L:O (luz:oscuridad) en una sala acondicionada con regulación de temperatura, humedad relativa y luminosidad.

\section{Bioensayos}

Los bioensayos se realizaron en una sala de crianza de 2,5 x 2,5 m, sin ventanas, con una puerta como único acceso, ubicada dentro del Laboratorio de Fitosanidad en la Estación Experimental Canchones. Para mantener las condiciones de temperatura, HR y fotoperíodo constantes los ensayos se realizaron en una cámara de crecimiento (Quimis, modelo Q-316M4, Brasil), siendo las condiciones de temperatura $25 \pm 0,5^{\circ} \mathrm{C}$ y fotoperíodo de $14: 10 \mathrm{~h}$ (L:O). En ensayos separados se evaluaron las diferentes humedades relativas relativas $(6,4 \%$, $9,2 \%, 35,0 \%, 37,1 \%$ y $80,0 \%$ ). Para asegurar un nivel constante de HR se siguió la metodología propuesta por De Courcy et al. (2004) que consistió en disponer los ácaros dentro de una cámara de acrílico transparente que en nuestro ensayo medía $10 \mathrm{~cm}$ (altura) x $15 \mathrm{~cm}$ (ancho) x $30 \mathrm{~cm}$ (largo). Las humedades relativas ensayadas fueron logradas utilizando sales higroscópicas las que no son tóxicas ya que no emiten elementos volátiles y cuya acción es absorber humedad. Las humedades de 6,4\%; 9,2\% y $37,1 \%$ fueron obtenidas como se describe en la Tabla 1. El 35,0\% HR (20,6 hPa), que correspondió
Tabla 1. Soluciones salinas saturadas usadas en los ensayos de eclosión con sus correspondientes humedades relativas (\%HR) y déficit de presión de vapor, DPV $(\mathrm{hPa})$ a $25^{\circ} \mathrm{C}$.

\begin{tabular}{lcc}
\hline Solución & HR $(\%)$ & DPV (hPa) \\
\hline $\mathrm{CaCl}_{2} \cdot 6 \mathrm{H}_{2} \mathrm{O}(50 \mathrm{~g})$ & 6,4 & 29,6 \\
$\mathrm{CaCl}_{2} \cdot 6 \mathrm{H}_{2} \mathrm{O}(10 \mathrm{~g})$ & 9,2 & 29,8 \\
$\mathrm{~K}_{2} \mathrm{SO}_{4}(50 \mathrm{~g})$ & 37,1 & 19,9 \\
\hline
\end{tabular}

HR, humedad relativa; DPV, deficit de presión de vapor.

a la humedad ambiente, se obtuvo al dejar abierta la cámara de acrílico dentro de la incubadora. Para el 80,0\% HR (6,3 hPa) se colocó en el piso de la cámara de acrílico una esponja totalmente humedecida y no se selló completamente, dejando un espacio de $3 \mathrm{~cm}$ para que esta no se empañara. La cámara de acrílico así dispuesta se colocó dentro de la incubadora. Previo a los ensayos, estos procedimientos fueron evaluados por tres días, para asegurar la constancia de la humedad relativa fijada, monitoreándose con un data logger (Keytag modelo 22r25). Una vez asegurada la constancia de las distintas humedades se procedió a montar los ensayos. Se depositaron cinco hembras del Neoseiulus sp. en una placa negra de acrílico de $6 \times 6 \mathrm{~cm}$ más un macho adulto (para asegurar la fertilización), dejándolas ovipositar por 24 horas para obtener huevos de edad conocida. Transcurrido ese tiempo se retiraron los adultos, dejándose 10 huevos por placa (unidad experimental), replicándose seis veces. Esto se hizo tanto para Neoseiulus sp. como para $P$. iorgius y para cada humedad relativa $(6,4 \%$, $9,2 \%, 35,0 \%, 37,1 \%$ y $80,0 \%)$. Posteriormente, las placas se pusieron en el interior de una cámara de acrílico transparente, esta se selló con vaselina sólida para evitar la modificación de la humedad relativa (De Vis et al., 2006). Separadamente, se evaluaron las diferentes humedades (tratamientos): $6,4 \%, 9,2 \%, 35,0 \%, 37,1 \%$ y 80,0\%. La eclosión de larvas o la deshidratación de los huevos se evaluó cada 24 h después del comienzo del experimento con una lupa estereoscópica Carl Zeiss Stemi SVG. En este proceso no fue necesario abrir la cámara de acrílico ya que las tapas eran transparentes.

\section{Análisis Estadístico}

El diseño experimental fue completamente al azar, con seis repeticiones por tratamiento, la unidad experimental fue una placa con 10 huevos. 
El porcentaje de eclosión de larvas, entre ambas especies de fitoseidos, se analizó mediante una prueba de $t$-Student. El porcentaje de eclosión de larvas intraespecie, se analizó mediante un ANDEVA a una vía $(\alpha=0,05)$. Previo a los análisis los porcentajes se transformaron angularmente $\left(\operatorname{arcosen} \sqrt{ } \mathrm{x} \%{ }^{-1}\right)$. Se aplicó la Prueba de Tukey $(\alpha=0,05)$ para separar las medias de los tratamientos. Para todos los análisis se utilizó el paquete estadístico InfoStat@

La humedad de eclosión media $\left(\mathrm{HR}_{50}\right)$ fue calculada mediante una regresión probit utilizando el programa estadístico MedCalc versión 16.4.3 (MedCalc Software bvba, Ostend, Bélgica; https:// www.medcalc.org; 2016). Esta regresión utilizada el estadístico chi-cuadrado $\left(\chi^{2}\right)$ de Pearson de la bondad de ajuste. Luego la ecuación (1), aplicada por Ferrero et al. (2010) se usó para calcular el $V P D_{50}$ (déficit de presión de vapor al cual el 50\% de las larvas eclosionan).

$V P D=6,112 \times e\left(\frac{17,67 \times T}{T+243,5}\right) \times\left(1-\frac{H R}{100}\right)$,

donde, $T$ es la temperatura. La $H R_{50}$ es a menudo discutida debido a su relación directa con la humedad relativa ambiental, en cambio la $V P D_{50}$ es dependiente de la temperatura, lo que permite comparaciones estandarizadas (Ferrero et al., 2010).

\section{Resultados y Discusión}

Los resultados de la influencia de las humedades relativas ensayadas sobre la viabilidad de los huevos de Neoseiulus sp. y P. iorgius a diferentes tiempos se presentan en la Tabla 2. Neoseiulus sp. a las $12 \mathrm{~h}$ no mostró diferencias significativas para las humedades evaluadas ya que no hubo eclosión de larvas a ningún nivel de humedad. En los siguientes períodos de evaluación hubo diferencias significativas entre las humedades relativas evaluadas, los porcentajes de eclosión para las 24 h oscilaron entre 3,33\% y $11,67 \%$, a las $36 \mathrm{~h}$ se obtuvo entre $33,33 \%$ y $61,67 \%$, a las $48 \mathrm{~h}$ los porcentajes fueron entre $40 \%$ y $98,33 \%$ y a las $60 \mathrm{~h}$ se obtuvo entre $50 \%$ y $100 \%$. Proprioseiopsis iorgius solo presentó eclosión de larvas a las $12 \mathrm{~h} \sin$ mostrar diferencias significativas para las humedades evaluadas; además no hubo eclosión de larvas a ningún nivel de humedad. En los siguientes períodos de evaluación hubo diferencias significativas entre las humedades relativas evaluadas

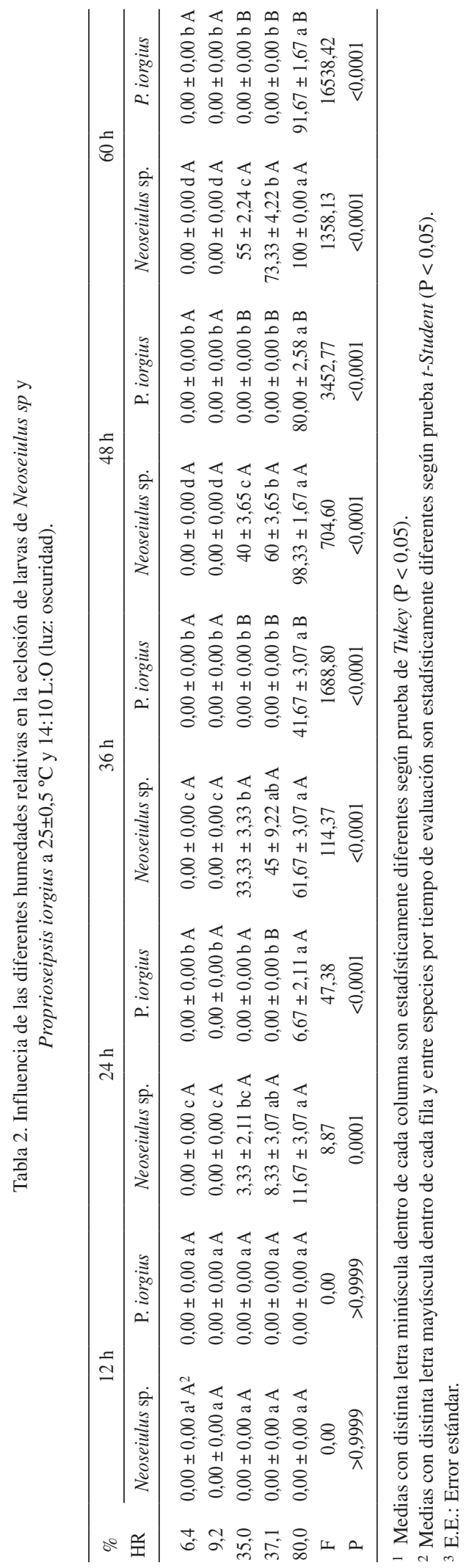


siendo el 80,0\% (6,3 hPa) el único nivel que permitió la eclosión de larvas, obteniéndose valores entre $6,67 \%$ y $91,67 \%$. Al comparar la eclosión de larvas entre ambas especies estas presentaron un comportamiento similar a las menores humedades relativas evaluadas $[6,4 \%(29,6 \mathrm{hPa})$ y $9,2 \%$ $(28,8 \mathrm{hPa})]$ con cero emergencias de larvas. A un $35 \% \mathrm{HR}(26,6 \mathrm{hPa})$, las diferencias se presentaron a partir de las $24 \mathrm{~h}$, produciéndose un incremento en la eclosión de larvas en Neoseiulus sp., al contrario de $P$. iorgius que tampoco a este porcentaje de humedad logró emergencia de sus larvas. Lo anteriormente descrito se repitió para el nivel de humedad de 37,1\% (19,9 hPa). Solo al 80,0\% HR $(6,3 \mathrm{hPa}) P$. iorgius logró emergencia de larvas, al compararlo con Neosiulus sp., se observa que a las $12 \mathrm{~h}$ y $24 \mathrm{~h}$ los porcentajes de emergencia fueron estadísticamente similares, pero a partir de las $36 \mathrm{~h}$ se presentaron diferencias significativas, logrando a las $60 \mathrm{~h}$ Neosiulus sp. un $100 \%$ de emergencia y P. iorgius un $91,67 \%$.

Con base en el traslape de los intervalos de confianza (IC) al 95\%, la humedad de eclosión media $\left(\mathrm{HR}_{50}\right)$ mostró diferencias significativas siendo Neoseiulus sp. el fitoseido más tolerante a una menor humedad relativa ya que con solo un $34,49 \%$ de HR (20,6 hPa de DPV) se obtuvo el 50\% de eclosión de sus larvas (Tabla 3). En cambio, P. iorgius, requiere un $72,36 \%$ de humedad relativa $(8,8 \mathrm{hPa}$ de DPV). Esto coincide con el hábitat en que ambos fitoseidos se distribuyen en la Región de Tarapacá. Neoseiulus sp. ocurre en la depresión intermedia, a nivel de pampa y oasis, en cambio $P$. iorgius habita la zona costera, requiriendo niveles altos de humedad. Karg (1976) cita a P. iorgius en el sector de Fray Jorge (3040'S 7140'W), Región de Coquimbo y Tello et al. (2011) lo encuentran en la costa de Iquique, Chile ((20'16' S 70 7' W). Los valores de $\mathrm{HR}_{50}$ y $\mathrm{DPV}_{50}$ están dentro de los obtenidos por otros autores para especies de fitoseidos (Tabla 4). Para el caso de Neoseiulus sp., la alta tolerancia de sus huevos a una menor humedad relativa expresada en su $\mathrm{HR}_{50}\left(\mathrm{DPV}_{50}\right)$ es similar a la mostrada por A. idaeus (=Cydnodromus picanus Ragusa) (30\%, 22,2 hPa) (Dinh et al., 1988) especie simpátrica con Neosiulus sp. en el norte de Chile. Por otro lado, la baja tolerancia de los huevos de P. iorgius a una menor humedad relativa expresada en su $\mathrm{HR}_{50}$ es similar a la mostrada por Typhlodromus lailae (Schicha) (71,1\%, 9,1 hPa) (Steiner et al., 2003a). La eficiencia de los controladores biológicos es a menudo afectada por condiciones locales tales como la humedad relativa baja. Esto es especialmente cierto para ácaros de la familia Phytoseiidae y particularmente para sus huevos (Ferrero et al., 2010). La tolerancia de los huevos a una baja humedad relativa en la familia Phytoseiidae puede relacionarse con el ambiente en el que han evolucionado sus especies (Negm et al., 2014). Al respecto, C. picanus, otro fitoseido colectado en el ambiente desértico de Pica por Ragusa (Ragusa, 2000) mostró una gran tolerancia a la baja humedad relativa con un $40 \%$ de eclosión de larvas a $42,35 \pm 5,01 \% \mathrm{HR}(23,7 \mathrm{hPa})$ y $29,44 \pm 1,47^{\circ} \mathrm{C}$ (Tello et al., 2009). Otro fitoseido muy tolerante a la baja humedad relativa es Typhlodromalus aripo De León, el cual registró un $70 \%$ de eclosión de sus larvas a $50 \% \mathrm{HR}(17,8 \mathrm{hPa})$ y con una temperatura de $27^{\circ} \mathrm{C}$ (Mutisya et al., 2014). En Sudamérica, el fitoseido Euseius citrifolius Denmark \& Muma ha demostrado una alta tolerancia de sus huevos a la baja humedad relativa, similar a Neoseiulus sp., registrando para 30 y $40 \%$ HR $(22,2$ y $19,0 \mathrm{hPa}$, respectivamente), porcentajes de eclosión de larvas de $30 \pm 10 \%$ y $57 \pm 7 \%$, a una temperatura de $25^{\circ} \mathrm{C}$ (De Vis et al., 2006). Steiner et al. (2003a) indican que alta humedad que requieren los huevos de $T$. lailae para eclosionar sugiere que esta especie podría ser utilizada en situaciones donde los huevos

Tabla 3. Humedad de eclosión media $\left(\mathrm{HR}_{50}\right)$ y déficit de presión de vapor medio $\left(\mathrm{DPV}_{50}\right)$ de huevos de Neoseiulus sp. y Proprioseiopsis iorgius a las $60 \mathrm{~h}$ de incubación, $25 \pm 0,5^{\circ} \mathrm{C}$ de temperatura y fotoperíodo de 14:10 h L:O (luz:oscuridad).

\begin{tabular}{|c|c|c|c|c|c|}
\hline \multirow{2}{*}{ Especie } & \multirow{2}{*}{$\mathrm{HR}_{50}\left(\mathrm{DPV}_{50}\right)^{1}$} & \multirow{2}{*}{ IC $95 \%$} & \multicolumn{3}{|c|}{ Pendiente } \\
\hline & & & \pm E.E. & $\chi^{2}$ & Gl \\
\hline Neoseiulus sp & $34,49 \%(20,7 \mathrm{hPa}) \mathrm{a}$ & $18,33-35,52(25,9-20,4 \mathrm{hPa})$ & $19,65 \pm 9,39$ & 4,38 & 3 \\
\hline P. iorgius & $72,36 \%(8,8 \mathrm{hPa}) \mathrm{b}$ & $69,99-74,83(9,5-8,0 \mathrm{hPa})$ & $29,39 \pm 0,47$ & $3.846,10$ & 3 \\
\hline
\end{tabular}

${ }^{1}$ Las humedades de eclosión media $\left(\mathrm{HR}_{50}\right)$ seguidas de letras iguales no son significativamente diferentes, basado en que los IC al 95\% se traslapan.; TE: tiempo de eclosión medio; IC: intervalo de confianza; E.E.: Error estándar de la media; $\chi^{2}$ : chi cuadrado; G.L.: grados de libertad. 
Tabla 4. Humedad de eclosión media $\left(\mathrm{HR}_{50}\right)$ y déficit de presión de vapor medio $\left(\mathrm{DVP}_{50}\right)$ de huevos de diferentes especies y cepas de fitoseidos a $25^{\circ} \mathrm{C}$ de temperatura.

\begin{tabular}{|c|c|c|c|c|}
\hline Fitoseido $^{1}$ & Temperatura $\left({ }^{\circ} \mathrm{C}\right)$ & $\mathrm{HR}_{50}(\%)$ & $\mathrm{DPV}_{50}(\mathrm{hPa})$ & Referencia \\
\hline A.idaeus $(\mathrm{CO})$ & 25 & 30,0 & 22,2 & Dinh et al. (1988) \\
\hline Neosiulus sp. (PI-CH) & 25 & 34,5 & 20,6 & Presente trabajo \\
\hline Phytoseiulus longipes Evans (PLLC-AR) & 25 & 43,0 & 18,2 & Ferrero et al. (2010) \\
\hline Typhlodromus athiasae Porath \& Swirskii (AR) & 25 & 43,0 & 18,1 & Ferrero et al. (2010) \\
\hline Neoseilus idaeus Denmark \& Muma (YC-AR) & 25 & 49,0 & 16,1 & Ferrero et al. (2010) \\
\hline P. longipes $(\mathrm{VA}-\mathrm{CH})$ & 25 & 50,0 & 15,9 & Ferrero et al. (2010) \\
\hline Amblyseius swirskii Athias-Henriot (RE-IS) & 25 & 63,0 & 11,4 & Ferrero et al. (2010) \\
\hline Neoseiulus californicus (McGregor) (CA-USA) & 25 & 65,6 & 10,9 & Walzer et al. (2007) \\
\hline N. californicus $(\mathrm{SI}-\mathrm{IT})$ & 25 & 66,3 & 10,7 & Walzer et al. (2007) \\
\hline N. californicus (CA-USA) & 25 & 66,9 & 10,5 & Walzer et al. (2007) \\
\hline N. californicus (TO-IT) & 25 & 67,0 & 10,5 & Walzer et al. (2007) \\
\hline N. californicus (CA-USA) & 25 & 68,2 & 10,1 & Walzer et al. (2007) \\
\hline N. californicus (HE-FR) & 25 & 68,5 & 10,0 & Walzer et al. (2007) \\
\hline Phytoseiulus macropilis (Banks) (YC-AR) & 25 & 69,0 & 9,8 & Ferrero et al. (2010) \\
\hline Phytoseiulus persimilis Athias-Henriot (K-BV) & 25 & 70,0 & 9,4 & Ferrero et al. (2010) \\
\hline N. californicus $(\mathrm{LC}-\mathrm{CH})$ & 25 & 70,2 & 9,4 & Walzer et al. (2007) \\
\hline N. californicus (VA-ES) & 25 & 70,3 & 9,4 & Walzer et al. (2007) \\
\hline T. lailae (AU) & 25 & 71,1 & 9,1 & Steiner et al. (2003a) \\
\hline P. iorgius (IQ-CH) & 25 & 72,4 & 8,8 & Presente trabajo \\
\hline Typhlodromis sessor De León (NJ-USA) & 25 & 80,0 & 6,3 & Sciarappa y Swift (1977) \\
\hline
\end{tabular}

${ }^{1}$ Origen de las cepas: Colombia (CO), Pica-Chile (PI-CH), Paso de Los Libres (Corrientes)-Argentina (PLLC-AR), Argentina (AR), Yapeyu (Corrientes)-Argentina (YC-AR), Valparaíso-Chile (VA-CH), Revadim-Israel (RE-IS), California-USA (CA-USA), Sicilia-Italia (SI-IT), Toscana-Italia (TO-IT); Hérault-Francia (HE-FR), Koppert BV (K-BV, cultivo masivo); La Cruz-Chile (LC$\mathrm{CH}$ ); Valencia-España (VA-ES); Australia (AU, cultivo masivo), Iquique-Chile (IQ-CH), New Jersey-USA (NJ-USA).

no estén sometidos a humedades extremadamente bajas y además la HR exceda el $70 \%$ durante varias horas en el día. De esta manera $P$. iorgius podría ser utilizado en condiciones ambientales de baja humedad, considerando que en la naturaleza los niveles de humedad rara vez permanecen constantes durante el día y los huevos podrían estar adaptados para tolerar estas fluctuaciones de humedad. Una estrategia planteada por Walzer et al. (2007) que permitiría reducir la pérdida de agua de los huevos sería el poseer un corion impermeable unido a una baja cantidad de agua en el huevo, esto debería conducir a bajas tasas de respiración. La tolerancia mostrada por Neoseiulus sp. a la baja humedad relativa debe ser evaluada experimentalmente a nivel de campo donde, de acuerdo a De Courcy et al. (2004), los efectos combinados de la morfología de la hoja, el movimiento del aire, la transpiración y la humedad ambiental crean un gradiente de humedad de la capa límite que puede proveer un microclima adecuado para los ácaros.

\section{Conclusiones}

Los huevos de Neoseiulus sp. presentan una alta tolerancia a la baja humedad relativa con un punto crítico $\left(\mathrm{HR}_{50}\right)$ de $34,5 \%$ (20,6 $\left.\mathrm{hPa}\right)$ que lo ubica dentro de los fitoseidos adaptados a condiciones de baja humedad. Por otra parte, P. iorgius, presenta una menor viabilidad de sus huevos en condiciones de baja humedad, registrando un punto crítico de $72,4 \%$ HR $(8,8 \mathrm{hPa})$ lo que lo sitúa en el extremo de los fitoseidos con menor tolerancia a la baja humedad relativa.

Para las condiciones de aridez extrema donde se desarrolla la agricultura en la Pampa del Tamarugal (Desierto de Atacama) es de suma relevancia poder disponer de fitoseidos que presenten tolerancia a la baja humedad relativa. Los resultados obtenidos para Neosiulus sp., sumado a futuras investigaciones sobre el efecto de la humedad en estadios móviles, consumo, parámetros de tabla de vida, respuesta funcional y numérica, entre otras, permitirá dilucidar el real potencial de este fitoseido como controlador biológico de plagas de tetraníquidos en el desierto del norte de Chile.

\section{Agradecimientos}

Los autores agradecen a la Universidad Arturo Prat y al Programa de Doctorado en Agricultura para Zonas Áridas-Desérticas de la Facultad de Recursos Naturales Renovables, por las facilidades dadas para el desarrollo de este estudio. 


\section{Literatura Citada}

Bakker, F.M.; Klein, M.E.; Mesa, N.C.; Braun, A.R. 1993. Saturation deficit tolerance spectra of phytophagous mites and their phytoseiid predators on cassave. Experimental and Applied Acarology, 17: 97-113.

De Courcy, W.; Kravar-Garde, L.; Fenlon, J.; Sunderland, K. 2004. Phytoseiid mites in protected crops: the effects of humidity and food availability on egg hatch and adult life span of Iphiseius degenerans, Neoseiulus cucumeris, N. Californicus and Phytoseiulus persimilis (Acari: Phytoseiidae). Experimental and Applied Acarology, 32: 1-13.

De Vis, R.M.J.; De Moraes, G.J.; Bellini, M.R.

2006. Effect of air humidity on the viability of predatory mites (Acari: Phytoseiidae, Stigmaeidae) common on rubber trees in Brazil. Experimental and Applied Acarology, 38 (1): 25-32.

Dinh, N.V.; Sabelis, M.W.; Janssen, A.

1988. Influence of humidity and water availability on the survival of Amblyseius idaeus and A. anonymus (Acarina: Phytoseiidae). Experimental and Applied Acarology, 4: 27-40.

Eaton, M.; Kells, S.A.

2009. Use of vapor pressure deficit to predict humidity and temperature effects on the mortality of mold mites, Tyrophagus putrescentiae. Experimental and Applied Acarology, 47: 201-213.

Ferrero, M.; Gigot, C.; Tixier, M.; Vanhouten, Y.; Kreiter, S. 2010. Egg hatching response to a range of air humidities for six species of predatory mites. Entomologia Experimentalis et Applicata, 135: 237-244.

Ghasemlooa, Z.; Pakyaria, H.; Arbaba, A.

2016. Cannibalism and intraguild predation in the phytoseiid mites Phytoseiulus persimilis and Typhlodromus bagdasarjani (Acari: Phytoseiidae). International Journal of Acarology, 42 (3): 149-152.

Ghazi, N.A.; Amano, H.

2016. The use of the cannibalistic habit and elevated relative humidity to improve the storage and shipment of the predatory mite Neoseiulus californicus (Acari: Phytoseiidae).

Guo, Y.; Lv, J.; Jiang, X.; Wang, B.; Gao, J.; Wang, E.; Xu, X. 2016. Intraguild predation between Amblyseius swirskii and two native Chinese predatory mite species and their development on intraguild Prey. Scientific Reports | 6: 22992 I DOI: $10.1038 /$ srep22992.

Hoy, M.A.

2011. Agricultural Acarology: Introduction to Integrated Mite Management. CRC Press, p. 407.

Hua, J.H.; Freed, S.; Wang, L.S.; Qin, W.J.; Chen, H.F.; Qin, H.G. 2014. Effect of temperature on development and reproduction of Proprioseiopsis asetus (Acari: Phytoseiidae) fed on asparagus thrips, Thrips tabaci. Experimental and Applied Acarology, 64(2): 235-44.

Karg, V.

1976. Zur kenntnis der Überfamilie Phytoseioidea Karg 1965. Zoologische Jahrbucher Systematik, 103: 505-546.
Lv, J.; Yang, K.; Wang, E.; Xu, X.

2016. Prey diet quality affects predation, oviposition and conversion rate of the predatory mite Neoseiulus barkeri (Acari: Phytoseiidae). Systematic and Applied Acarology, 21 (3): 279-287.

Mutisya, D.; El-Banhawy, E.M.; Kariuki, CH.W.; Khamala, C.P.M. 2014. Typhlodromalus aripo de Leon (Acari: Phytoseiidae) development and reproduction on major cassava pests at different temperatures and humidities: an indication of enhanced mite resilience Acarologia, 54 (4): 395-407.

Negm, M.W.; Alatawi, F.J.; Aldryhim, Y.N.

2014. Biology, predation and life table of Cydnoseius negevi and Neoseiulus barkeri on the old world date mite, Oligonychus afrasiaticus. Journal Insect Science, 14: 1-6.

Pozzebon, A.; Loeb, G.M.; Duso, C.

2015. Role of supplemental foods and habitat structural complexity in persistence and coexistence of generalist predatory mites. Scientific Reports | 5:14997 IDOI: 10.1038/ srep14997.

Ragusa, S.

2000. A new Cydnodromus (Parasitiformes, Phytoseiidae), from the desert of the Northern Chile. Phytophaga (Palermo), 10: 3-10.

Sciarappa, W.J.; Swift, F.C.

1977. Biological studies of Typhlodromus sessor (Acarina: Phytoseiidae). Annual Entomology Society American, 70: 285-288.

Steiner, M.; Goodwin, S.; Wellham,T.; Barchia, I.; Spohr, L. 2003a. Biological studies of the Australian predatory mite Typhlodromalus lailae (Schicha) (Acari: Phytoseiidae). Australian Journal of Entomology, 42: 131-137.

Tello V.; Vargas. R.; Araya. J.; Cardemil A.

2009. Biological parameters of Cydnodromus picanus and Phytoseiulus persimilis raised on the carmine spider mite Tetranychus cinnabarinus (Acari: Phytoseiidae, Tetranychidae). Ciencia e investigación agraria, 36 (2): 277-290.

Tello, V.; Briceño, R.; Castillo, P.

2011. Parámetros biológicos de Proprioseiopsis iorgius sobre Tetranychus desertorum (Acari: Phytoseiidae, Tetranychidae). Revista Colombiana de Entomología, 37 (1): 62-66.

Van Dinh, N.; Sabelis, M.W.; Janssen, A.

1988. Influence of humidity and water availability on the survival of Amblyseius idaeus and A. anonymus (Acarina: Phytoseiidae). Experimental and Applied Acarology, 4: 27-40.

Van Leeuwen, T.; Van Pottelberge, S.; Nauen, R.; Tirry, L.

2007. Organophosphate, insecticides and acaricides antagonise bifenazate toxicity through esterase inhibition in Tetranychus urticae. Pest Management Science, 63: 1172-1177.

Walzer, A.; Castagnoli, M.; Simoni, S.; Liguori, M.; Palevsky,

E.; Schausberger, P.

2007. Intraspecific variation in humidity susceptibility of the predatory mite Neoseiulus californicus: Survival, development and reproduction. Biological Control, 41: 42-52. 
